\title{
Fisuras en los discursos de la intervención social contemporánea $^{1}$
}

\author{
Ana Lucia Paz Rueda y Viviam Unás Camelo
}

\begin{abstract}
Despite the importance of social intervention in today's world, reflections about its nature are not frequently found. This article discusses the implications for social intervention that arise from the discourses regarding an "intervened subject" given his/her excluded condition. In order to do this, the article describes the circumstances and contexts in which exclusion appears and three forms this condition takes in social intervention discourses. The article also explains some theoretical fissures in these discourses, which invite discussion and reflection on the real impact of social intervention as a vehicle for social change.
\end{abstract}

\section{Los actores de la intervención social contemporánea}

¿Qué reconoce la intervención hoy como sujeto intervenido? ¿Cuáles son sus características, en términos de carencias o necesidades, que implican un ejercicio de asistencia e intervención social? Para responder a estos interrogantes se hace imprescindible determinar algunas características de la Intervención Social Contemporánea (ISC) y de los actores que la constituyen porque ellos indican la forma en que son construidos conceptualmente los sujetos intervenidos. Esto implica distinguir

\footnotetext{
${ }^{1}$ Este artículo es producto de un trabajo de investigación, adelantado por el grupo de investigación sobre "Intervención social” que pertenece al Centro de Investigaciones en Estudios Sociales y Jurídicos, CIES, del Departamento de Humanidades y Ciencias Sociales de la Universidad ICESI. En la recolección de datos que hicieron posible este artículo participaron activamente los profesores José Darío Sáenz y Enrique Rodríguez. La investigación ha arrojado como resultado un balance revelador de la forma como la literatura y las ciencias sociales abordan el problema de la intervención social. La mayor parte de los textos revisados se centraban en el metodología y en la evaluación de procesos, sólo un pequeño número de éstos tenía un carácter reflexivo sobre la intervención como objeto de estudio de las ciencias sociales. Este hecho resulta paradójico si se considera la importancia creciente de la intervención como escenario laboral para sociólogos, antropólogos y otros científicos sociales y si se reconoce la importancia que la intervención tiene como lugar en el que se producen, hoy por hoy, muchas de las tensiones que competen a las transformaciones del Estado, del mundo social y de los sujetos en su calidad de ciudadanos. Este documento constituye sólo una aproximación sociológica a la reflexión sobre los sujetos intervenidos y la exposición de algunos interrogantes sociológicos aún por resolver.
} 
la ISC de otras formas de asistencia social que, como las concepciones y prácticas de la beneficencia pública (efectuadas por instituciones estatales) o la filantropía social (ejecutadas por sectores dominantes) pretenden aliviar la pobreza y la mendicidad. La ISC, en cambio, responde a una política pública (una acción sobre el ámbito de lo público) que, adelantada desde el Estado o desde la sociedad civil, busca generar seguridad social extendida a todos los ciudadanos en la forma de derechos sociales y públicos. Es imprescindible reconocer que las intervenciones y las políticas sociales tienen estrecha relación con los paradigmas socioeconómicos y políticos derivados del neoliberalismo y el Estado de Bienestar. ${ }^{2}$

Esta relación entre intervención y política pública permite pensar la acción de intervenir como la respuesta a la dificultad de un sistema social complejo para resolver efectivamente necesidades y problemas, es decir, para autorregularse. La complejidad de los sistemas sociales indica que en los procesos de autorregulación participan diversos sectores y ámbitos, por lo que la intervención debe idealmente reconocer la necesidad de operar sobre escenarios micro o macro. ${ }^{3}$ En el primer caso, se atienden necesidades de los sujetos de manera personalizada, a partir de modelos e ideologías de prestación de servicios profesionales, mientras que en el segundo caso se trata de un esfuerzo institucional que, además de modelos e ideologías de servicios, involucra formas de organización social, legal, filosófica y política que confluirían en transformaciones sociales amplias.

Los ámbitos micro y macro en los que opera la intervención demandan la presencia de tres actores sustantivos: el sistema político, la profesión como representación del mundo académico y de las ONG y, en tercer lugar, la población intervenida. El primer actor mencionado, el Estado, representa al sistema político, sus funciones se concentran en gestionar y orientar la intervención a través de líneas de trabajo en política social que velen por el bienestar de la comunidad (Gough, 1982). En la confluencia entre políticas sociales y bienestar de la comunidad se presentan dos formas visibles: la provisión

\footnotetext{
${ }^{2}$ La revisión documental efectuada permite afirmar que las intervenciones sociales están orientadas por objetivos sociales amplios que buscan afectar colectivos y que se relacionan con los modelos de desarrollo de una sociedad, bien sea como apoyo o como crítica a ellos. Esto indica que el nexo de los estados sociopolíticos con el mercado, y con las formas políticas mismas, afectan todas las dimensiones de la intervención social. No es objeto de este artículo realizar una discusión de las características del Estado de Bienestar o del neoliberalismo. Se consideran, por lo tanto, dadas unas ciertas características ligadas al contexto socioeconómico que son inevitables y que son registradas por distintos autores como Castel (1997), Sennett (2003), Touraine (1969, 1986), Corvalán (1996), Carballeda (2001), Ian Gough (1982) entre muchos otros autores revisados para la elaboración de este documento.

${ }^{3}$ Al respecto ver: Sánchez Vidal, Alipio, Ética de la intervención social, Ed. Paidós, Barcelona, 1999.
} 
estatal de servicios sociales a través de la entrega de recursos y la ejecución directa o contratada de proyectos, planes o programas.

Los segundos actores en mención, los profesionales en ciencias sociales, intervienen, bien conceptualizando sobre el tema (mundo académico) o bien ejecutando proyectos (ONG). En estos casos se trata de formas de reglamentación estatal de actividades privadas, ejercidas por individuos o corporaciones, que directamente alteran las condiciones inmediatas de vida de los individuos y grupos dentro de una población y que, más que "mejorar”, afectan la situación en términos cuantitativos y cualitativos.

La ISC contemporánea, si bien depende en buena medida del sistema político no siempre es ejecutada por éste. Una forma extendida de ejercicio de la intervención social no estatal proviene de las Organizaciones no Gubernamentales (ONG). Éstas surgen, entre otras cosas, ante al paulatino desmantelamiento de las instituciones que en el pasado administraron la asistencia social y se configuran como un modo de actividad política, en tanto buscan incidir en lo público, pero desde la acción privada. En este sentido las ONG difieren y disputan ciertos espacios públicos con actores sociales de mayor tradición como los partidos políticos, los grupos de presión, los grupos de interés y con otras formas de acción social como los movimientos sociales. ${ }^{4}$

Cuando los profesionales no están incluidos en ONG sino en el mundo académico, se relacionan con el trabajo de establecer estándares, conceptualizaciones y, en algunos casos, coordinar la ejecución y evaluación de procesos de intervención. En este sentido, la ISC se diferencia de las formas filantrópicas o de beneficencia pública en tanto está regida por actividades técnicas $y$, por tanto, por una relación directa con el conocimiento científico, materializado en habilidades metodológicas y técnicas para diseñar organizar, realizar y evaluar procesos.

Ambos actores, Estado y profesionales vinculados desde ONG o la acadamia, permiten pensar la Intervención Social Contemporánea como una acción organizada frente a problemáticas sociales no resueltas. Esta condición de acción organizada supone a la intervención como un proceso de orden racional, pues se funda en una

\footnotetext{
${ }^{4}$ La posibilidad de accionar político de las ONG, sin embargo, se ve amenazada por su compleja relación con el Estado y el mercado de proyectos sociales. Las ONG se plantean a sí mismas como instituciones sin ánimo de lucro que operan al margen de las disposiciones gubernamentales. Esta idea fundante se juega en medio de un mercado competitivo, con importante fuente de financiación y flujo continuo de capitales, dada la enorme cantidad de recursos económicos destinados a la ejecución de proyectos sociales, particularmente en la ciudad de Cali. C.f. Sáenz, José Darío, “Temas de reflexión en la intervención social”, publicado en este mismo número.
} 
intención manifiesta de modificar o transformar una situación social que se considera indeseable e injusta, ante todo para el grupo que la padece. Es con respecto a este último punto en el que entra en juego el tercer actor referenciado: la población intervenida. Lo que se reconoce como población intervenida constituye el sector social que se determina destinatario de la intervención, titular de los problemas a resolver y encargado de delegar autoridad tanto en el Estado como en profesionales de ONG y académicos. Las relaciones entre Estado, profesionales y población intervenida pueden estudiarse desde múltiples perspectivas. En este caso interesa reconocer cómo la forma en que se piensa el "sujeto intervenido", y su relación con la exclusión social, potencia principios y lógicas de intervención concretas. Para tal fin es necesario reconocer al "sujeto intervenido” como el resultado de una construcción retórica y de una serie de acciones por medio de las cuales la intervención social identifica y configura técnica y socialmente a ciertos sectores sociales como excluidos. Los discursos sobre el sujeto intervenido estarían hablando entonces de la lectura que la ISC y los actores que intervienen, incluyendo al sector académico, hacen sobre la exclusión social y, también, de los modos en que se concibe la superación o contención de ésta.

Este trabajo de lectura y construcción sobre la exclusión afecta a su vez el mundo social: impacta subjetivamente a los sujetos que participan en la intervención en tanto modela subjetividades, inventa e imagina la legitimidad que distribuye el poder, orienta demandas sociales y se convierte en un medio de estigmatización, control social y dispositivo para la acción colectiva. En otras palabras, la intervención social interviene no sólo desde sus resultados sino también desde su producción retórica: el mundo social absorbe conceptos de las ciencias sociales y de esta manera se dibujan fronteras visibles entre unos sujetos excluidos y otros que no lo son, lo que termina mediando las relaciones entre actores.

Se advierte entonces que los actores que participan de la intervención social pretenden abordar el problema de la exclusión social. Estas condiciones responden a un contexto histórico que las determinan y las transforman: los sujetos excluidos lo han sido por causas y síntomas diferentes, aunque significativamente asociados, a lo largo de la historia de la asistencia social. Estudiar la condición de “intervenido" pasa entonces por identificar los contextos que modelan la exclusión que permiten a la intervención establecer objetivos para su superación. El siguiente aparte pretende describir y sustentar la idea de que lo que se reconoce hoy como "sujeto intervenido" es la condición de ciertos sectores sociales, irrepresentables en una clase social, que se 
hayan excluidos de redes que posibiliten acceso al mundo del trabajo y a los circuitos de consumo. En otras palabras, se describirá cómo la situación de exclusión que atiende la ISC corresponde a la situación de ciertos sujetos y sectores sociales que, dada su marginalidad con respecto a vínculos y redes de relación fuertes, se ubican en escenarios de limitada movilidad y precarias posibilidades de ejercer una ciudadanía plena.

\section{El sujeto intervenido como sujeto excluido}

La exclusión se entiende como la imposibilidad de insertarse a redes sociales. Para ilustrar la relación entre exclusión social y red de vínculos sociales es necesario establecer una diferencia sustantiva en la función que las redes de relación cumplen en el mundo contemporáneo con respecto a otros momentos históricos. Tradicionalmente, las redes sociales han permitido a los sujetos establecerse, esto es, desarrollar arraigos culturales, proyectos vitales y dinámicas de inserción al mundo del trabajo que se fundan en el sostenimiento y la experiencia acumulada en un escenario específico. Esto explica cómo las condiciones de desafiliación, del siglo XIV por ejemplo, se manifestaban en la movilidad constante del vagabundo quien, al no contar con vínculos y redes estables, se veía obligado al nomadismo y la errancia. Consecuentemente con esta situación, la asistencia social y los órganos de control se encargaban de castigar o atender el vagabundeo con estrategias que procuraran inmovilidad ya fuera dentro de instituciones o territorios concretos. ${ }^{5}$

La inestabilidad del mundo del trabajo transforma hoy visiblemente la función de los vínculos y redes sociales. Si bien éstos continúan siendo medios claves en los procesos de afiliación, su fortaleza no depende ya tanto de su capacidad para brindar escenarios vitales sólidos, sino de su ubicación estratégica como lugares de movilidad que permitan responder a las crecientes demandas de flexibilidad. Esto explica porqué la desafiliación contemporánea se manifiesta en inmovilidad, no sólo laboral sino también política, social y comunitaria y permite entender porqué los procesos de ISC se interesan cada vez más en el trabajo de fortalecimiento de redes y vínculos sociales que proporcionen autonomía a los sujetos.

\footnotetext{
${ }^{5}$ Castel, Robert, Las Metamorfosis de la cuestión social. Una crónica del salariado, Paidós, Buenos Aires, 1997.
} 
Para comprender con mayor profundidad cómo funciona la relación entre vínculos sociales y procesos de exclusión conviene detenerse en la noción de sujeto incluido. Existen dos formas de comprender el estatuto del sujeto integrado. Una lo relaciona con el conjunto de condiciones que permiten a cierto sector participar de una ciudadanía plena $^{6} \mathrm{y}$, la otra, en algunos casos complementaria, con las condiciones que permiten a ciertos sujetos participar activamente de los circuitos de consumo. En ambos casos, sin embargo, se está aludiendo a la integración como la situación de ciertos sujetos que, dado su cumplimiento de deberes sociales, están en posibilidad de acceder a derechos ciudadanos o a beneficios dentro del sistema social. En otras palabras, se está anunciando la relación directa entre la inclusión, como el resultado de la inserción al mundo del trabajo, el acceso a derechos y la integración a circuitos de consumo.

Bajo la noción de mundo conexionista, ${ }^{7}$ esta inclusión e inserción social está dada por la acumulación y uso estratégico del capital social, entendido como conjunto y calidad de las relaciones sociales. No es extraño entonces que los sujetos incluidos desplieguen buena parte de su energía en la gestión de redes sociales que permiten su movilidad en el espacio y garanticen su supervivencia en el mundo del trabajo. Ante el debilitamiento institucional, provocado en parte por el debilitamiento del Estado de Bienestar, las redes de vínculos soportan al sujeto ya que le permiten insertarse. ${ }^{8}$

En los procesos de inserción intervienen distintos factores, entre ellos la personalidad. ${ }^{9}$ La puesta en juego de la personalidad entendida como las competencias y disposiciones de los sujetos para producir vínculos sociales, cumple un papel determinante porque impacta los procesos de intervención social y sus discursos al menos en tres sentidos: en primer lugar integra al ejercicio de intervención la promoción de autonomía, entendida como el trabajo sobre competencias, disposiciones y cualificación en la autogestión de redes, y en este sentido los discursos se concentran en promover la cualificación y autogestión para una inserción positiva. En segundo lugar,

\footnotetext{
${ }^{6} \mathrm{Al}$ respecto ver: Carballeda, Alfredo, La intervención en lo social. Exclusión e integración en los nuevos escenarios sociales, Paidós, Buenos Aires, 2002.

${ }^{7}$ Propuesta en: Boltansky, Luc, El nuevo espíritu del capitalismo, Ed. Akal, Madrid, 2000.

${ }^{8}$ Esto atañe no sólo a los sujetos o grupos sociales sino también a la economía en tiempos globalizados: los países poderosos desplazan capitales a un país pequeño (pero tienen entera libertad para retirarlo cuando quieran). El país afectado, por su parte, no cuenta con esa movilidad. Necesita ese dinero para su desarrollo y la retirada abrupta de capitales puede desencadenar una crisis. Así mismo asegura Boltanski que las "relaciones de explotación basadas en diferenciales de movilidad parecen innumerables: mercados financieros versus países; mercados financieros versus empresas; multinacionales versus países; experto mundial vs. empresas; empresa versus personal precario; consumidores versus empresa". $\mathrm{Al}$ respecto ver: Boltansky, Luc, Op. Cit.

${ }^{9}$ Sennett, Richard, El respeto. Sobre la dignidad del hombre en un mundo de desigualdad, Anagrama, Barcelona, 2003.
} 
compromete los procesos de intervención con el trabajo de fortalecimiento de vínculos sociales; se habla entonces de la necesidad inminente de vincularse a redes como vehículos de inserción. En tercer lugar, determina al sujeto intervenido en virtud de su capital social y su condición de exclusión, por tanto segmenta los grupos sociales y los interviene de acuerdo al tipo de exclusión que presentan.

Los tres discursos coinciden en su relación con aquellos sujetos que no consiguen conectarse eficientemente con redes, lo que se expresaría en marginación del mundo laboral y de los procesos de intercambio económico. La ISC atendería pues a los “otros”, a los que no pueden circular, a aquellos cuya inmovilidad les impide acceder a derechos fundamentales. En palabras de Castel, "excluidos serían aquellos que han visto romper sus vínculos con los demás, aquellos que han sido enviados a los márgenes de la red, allí donde se pierde toda visibilidad, toda necesidad y, prácticamente toda existencia”. ${ }^{10}$

Los excluidos son irrepresentables en tanto no constituyen una clase social. La exclusión constituiría un término ilusoriamente neutro: los excluidos lo son, en apariencia, sin responsabilidad de ninguna otra figura social que no sea la de su propia trayectoria biográfica, su carácter, sus competencias y cualificación. Serían entonces “(aquellos) a los que nadie representa, a los que, abandonados también por las instancias críticas nacidas de las luchas obreras, han quedado relegados a la asistencia, humillante e ineficaz”. ${ }^{11}$ Conforman, más bien, una falla aparente del tejido social por lo que se tiende a hablar de excluidos más en términos de proceso y problemática que de grupos sociales: se habla de exclusión más que de excluidos y de marginación más que de marginados. En definitiva, esta noción de exclusión - a diferencia del modelo de clases - indica una categoría social que no se relaciona aparentemente con aspectos estructurales. Sin embargo, es posible establecer relaciones que superan esta limitante. En principio es necesario develar los mecanismos en que la inclusión de unos sujetos sociales depende de la exclusión de otros. Castel, por ejemplo, sostiene que no hay que olvidar que la movilidad de los incluidos puede darse en tanto se soporta en la inmovilidad de los que no lo son. ${ }^{12}$ Los excluidos, o integrados con menor poder de movilidad, se constituyen en puntos de red fijos que permiten a los integrados

\footnotetext{
${ }^{10}$ Castel, Robert, Las Metamorfosis de la cuestión social. Una crónica del salariado, Paidós, Buenos Aires, 1997. (p.) 447.

${ }^{11}$ Boltanski, Luc, El nuevo espíritu del capitalismo, Ed. Akal, Madrid, 2000.

12 Castel, Robert, Las Metamorfosis de la cuestión social. Una crónica del salariado, Paidós, Buenos Aires, 1997. (p. 466 y ss.)
} 
incrementar su facultad de moverse dentro de redes. Sin dichos puntos de red los integrados tendrían dificultades para moverse pues "a medida que se desplazan, perderían tantas relaciones como fueran creando. No podrían acumularlas. El capital se les escaparía". ${ }^{13}$ De esta manera, mientras algunos sujetos o grupos sociales más fuertes se mueven y navegan en redes, otros, más débiles, se insertan precariamente en escenarios más frágiles y provisorios: empleos de corta duración o de condiciones inseguras, proyectos a corto plazo, desempleo definitivo entre otras múltiples opciones.

La dificultad para la inserción social de los excluidos lleva a reflexionar sobre un segundo aspecto: la inclusión en redes sociales. La dificultad para insertarse se incrementa si se comprende que entre más tiempo un sujeto se mantenga en situación de precariedad o exclusión, más tiempo tardará en incluirse, en cualificarse técnicamente, en adquirir bienes y definir proyectos de vida a largo plazo. La precarización precariza a su vez la vida misma de los sujetos y condiciona las posibilidades de integrarse, en otras palabras, inmoviliza. La inmovilidad hace referencia entonces a la restricción para recrear y producir vínculos sociales. Un sujeto excluido ha sido despojado, entre otras cosas, de la esperanza de ampliar sus relaciones con otros, en procura no sólo de la supervivencia en el trabajo sino también de producción de proyectos colectivos y políticos. Y es esta esperanza de movilidad e inserción la que pretenden concretar los proyectos que buscan el fortalecimiento de tejidos sociales, como dispositivos de desarrollo y fortalecimiento del poder de participación comunitaria.

Por otra parte, un tercer elemento permite analizar cómo la intervención social se concentra hoy en la contención y resolución de la exclusión a través de la labor de promoción de la autonomía y la inserción o integración social. Esto sugiere una relación entre autonomía e integración. Esta relación pareciera contener una tensión interna: cuando se alude a integración a redes, se habla, de fondo, de procesos que implican un tipo particular de pertenencia y dependencia de vínculos y tejidos sociales. Por su parte, la autonomía nombra una suerte de independencia y capacidad de gestión al margen o por encima de disposiciones sociales. Parecería paradójico entonces que autonomía e integración se anuncien como procesos complementarios. Las continuidades que se tejen en la relación entre uno y otro proceso son complejas e invitan a una exposición más detallada. Para este efecto es necesario advertir que cuando se menciona la

\footnotetext{
${ }^{13}$ Castel, Op. Cit. p. 472.
} 
importancia central de los vínculos, redes y tejidos sociales para el acceso a derechos ciudadanos, no necesariamente se está hablando en perspectiva de proyectos colectivos. Para el sujeto liberal los vínculos se traducen hoy en oportunidad individual y no necesariamente en horizonte común. La rentabilidad de los procesos de inserción a redes sociales, por ejemplo, se materializa en el éxito y sostenimiento en el mundo del trabajo. Las redes potencian entonces los procesos de individuación: implican desapego, movilidad y trashumancia. Sin instituciones fuertes que acojan y asistan a los sujetos sociales, el mundo social invita a la formación de tejidos en los que se despliega la incertidumbre del tránsito y la movilidad. El sujeto que habita, o más bien navega, por redes sociales se concreta en un tipo particular de individualismo-red: un individualismo que usa los vínculos como oportunidades de autogestión.

Los discursos y métodos destinados a fortalecer vínculos sociales y promover la autonomía se enfrentan entonces al reto de propiciar la integración de los sujetos excluidos. Sin embargo, en este proceso la intervención social tiene un impacto limitado. A continuación se retomarán algunos elementos de los discursos contemporáneos sobre intervención social ya presentados, se ampliarán otros y se señalarán, a modo de fisuras, algunos cuestionamientos que pueden efectuarse a dichos discursos que, desde la necesidad de integrar a los sujetos excluidos, restringen su potencial como motor de cambio.

\section{Fisuras en los discursos contemporáneos de intervención social ${ }^{14}$}

En esta sección se retomarán los tres discursos centrales, ya expuestos, que orientan el trabajo de intervención sobre sujetos excluidos (la lógica de promoción de la autonomía, el discurso sobre el fortalecimiento de vínculos y las prácticas orientadas desde la focalización y fragmentación de la población intervenida en función de su exclusión) para describir cinco fisuras, entendidas como fallas o debilidades, contenidas en estos discursos. El término fisura denota una fuga teórica no resuelta, un

\footnotetext{
${ }^{14}$ Si bien para efectos expositivos en este documento las fisuras y sus consecuencias se describen de manera independiente, lo cierto es que las tensiones y hechos que las componen se presentan en el mundo social como hechos interdependientes, relacionados entre sí y con causas y efectos comunes.
} 
algo que se escapa dentro de las construcciones retóricas de la intervención. El uso de este término permite hacer visibles dos procesos simultáneos: por una parte interroga a la intervención desde lo no expresado o no considerado en sus acciones y, por otro, esboza lugares de estudio y propuestas que pueden ser apropiadas por las ciencias sociales en el camino de construir una intervención social más eficiente. El esquema general de la argumentación se resume en el siguiente gráfico:

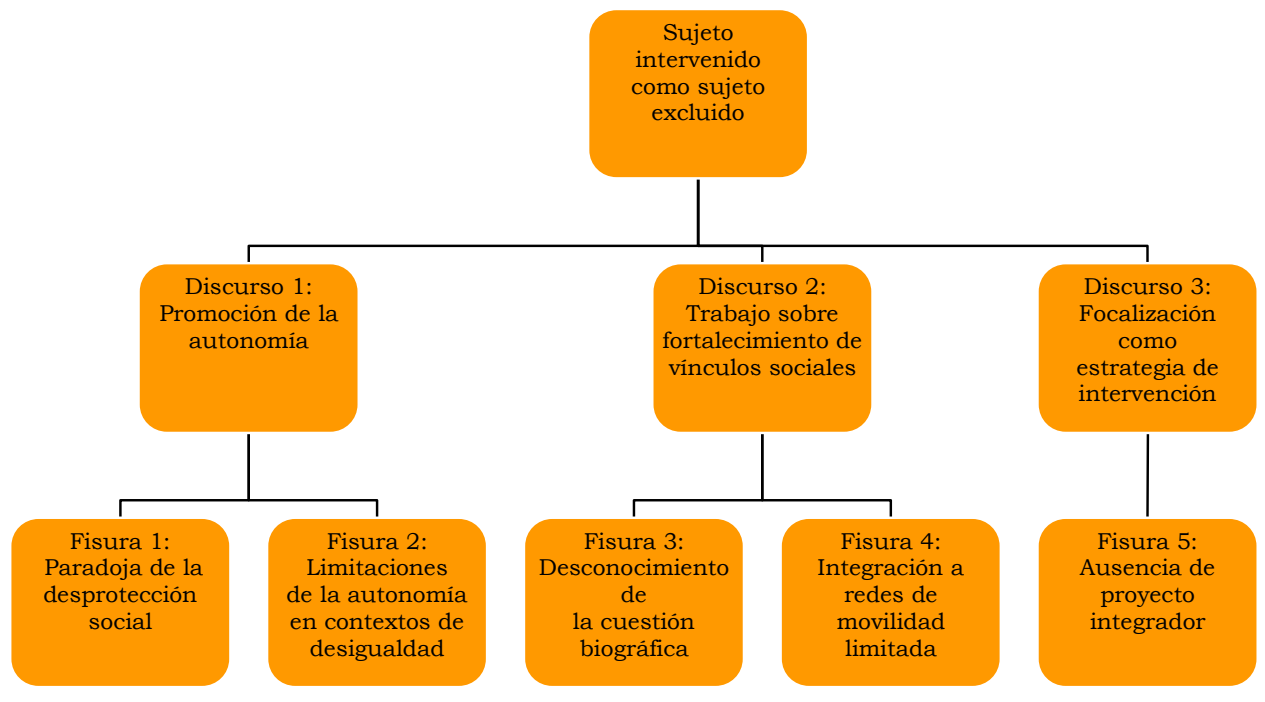

\section{Discurso 1 \\ Promoción de la autonomía}

Fisura 1: La paradoja de la desprotección social

En los proyectos de intervención, la relación entre fortalecimiento de vínculos sociales y autogestión de redes sociales se manifiesta en la exigencia que se le hace a los sujetos excluidos para que se tornen autónomos, al punto de que muchos planes y programas deciden apoyar sólo a comunidades que, en tiempos de crisis, hayan logrado diseñar, soñar o sostener un proyecto. En términos neoliberales, se espera apoyar 
comunidades que sean capaces de sumarse al libre juego de las fuerzas sociales como una más que compite y participa de la sociedad de mercado. Es ésta una fuerza que se plantea despolitizada, en tanto no se representa en partidos ciudadanos y más bien se promueve como forma autorregulada de lograr desarrollo comunitario. Así, la promoción de competencias y capacidad para autogestionar proyectos de movilidad e integración a redes pretende hacer a los sujetos menos dependientes de la asistencia social. Esto influye en que se considere la dependencia a la asistencia social no como un derecho ciudadano, sino como una condición que expresa carencias. Los que dependen de la gestión de instituciones y prácticas de asistencia son los marginados que, dada su exclusión del mundo del trabajo y los circuitos de consumo, tienen cada vez menos posibilidades de procurarse derechos y garantizarse su seguridad social básica. En oposición, ser independiente pasa por procurarse una cierta emancipación de la protección estatal: los incluidos son independientes y, por tanto, responsables de su seguridad y atención. Los programas que promueven la integración de los excluidos estarían promoviendo de fondo un proceso de desresponsabilización del Estado de su función como garante del bienestar de los sujetos sociales. Protección social y autonomía emergen entonces como dos términos en tensión. La fisura descrita invita a repensar el término autonomía y las acciones que la desarrollan en clave de un interrogante básico: ¿Cómo lograr una intervención que no se convierta en tutelaje y una independencia que no se traduzca en desprotección?

Fisura 2: Las limitaciones de la autonomía en contextos de desigualdad

Una segunda ruptura y contradicción a la que está sujeta la promoción de la autonomía se relaciona con los actores que participan como agentes o como receptores de los procesos de intervención. Los interventores actúan frente a receptores de las estrategias de intervención, dada una doble condición: son portadores de un problema determinado o son deficitarios sociales o ambos. ${ }^{15}$ Esto remite necesariamente a un rol que se ejerce desde la carencia y frente al cual el interventor actúa y se relaciona con el sujeto de intervención. En el caso de los portadores de problemas se reconoce una suerte

15 Corvalán, Javier, "Los paradigmas de lo social y las concepciones de intervención en la sociedad”, Tesis de Doctorado, Departamento de Ciencias Políticas y Sociales, Universidad Católica de Lovaina, 1996. 
de potencial colectivo para la acción mientras en los deficitarios sociales la limitación es aún mayor, lo que los distingue de la clase obrera y popular. En ambos casos los actores desaparecen: como portadores se subsumen en un concepto amplio y ambiguo y como deficitarios se diluyen en unas características grupales que no estructuran actores ni explicitan roles. Esto ratifica la relación de subordinación de los intervenidos frente a sus interventores, lo que indica que el trabajo de intervención se efectúa en el encuentro entre dos grupos sociales desiguales: uno beneficiario de servicios y, por lo tanto, vulnerable, y otro interventor y en apariencia no vulnerable. Esta situación de desigualdad se fortalece, como se ha mencionado previamente, cuando se nombra al otro, al intervenido, no desde su lugar productivo sino desde su carencia (no se habla, por ejemplo, de niños sino de menores, lo que traduce en "carentes de protección adulta”). Así, se alude a desfavorecidos, vulnerables, necesitados y carentes, lo que termina afectando la ubicación de éstos como sujetos de demanda y autonomía. A esto se suma el hecho de que en el campo de la intervención, considerando su posición como vigoroso mercado de proyectos sociales, la población se convierte en un tipo de recurso; los sectores intervenidos pueden en ocasiones convertirse en disputados consumidores de la oferta de programas más que en sujetos de derechos políticos y sociales. ${ }^{16}$ Pensar a los intervenidos como consumidores de programas y a la población intervenida como recurso lesiona las posibilidades de construir una autonomía ciudadana plena, entendida ésta, como el ejercicio de deberes y el uso de derechos que no deberían restringirse a la condición de "beneficiario" temporal de proyectos de intervención social.

Por otro lado la promoción de autonomía en contextos de desigualdad puede favorecer la producción de relaciones de dependencia en tanto los agentes interventores aparecen como los que proveen recursos para el desarrollo de acciones participativas y “autogeneradas”. Esto tiene como consecuencia que los recursos distribuidos sean percibidos más como donaciones que como derechos indisociables de la condición de ciudadano. A esta situación contribuyen tres razones básicas: en primer lugar, el recurso obtenido no parece disponible para el conjunto de ciudadanos. En segundo lugar, no se percibe su recepción como resultado de una responsabilidad estatal. En tercer lugar, el recurso no es derivado de la condición de ciudadano sino de un trabajo de “discriminación positiva” en el que la intervención selecciona técnicamente a

\footnotetext{
${ }^{16}$ Esto es visible en la adopción por parte de los procesos de ISC de una retórica empresarial que alude a autogestión, formación de alianzas estratégicas, competencias sociales. Estos discursos terminan elaborando, en procura de autonomía, un estatuto de sujeto intervenido como "beneficiario” o “cliente”.
} 
determinados sujetos como excluidos. En definitiva, los sujetos intervenidos, a los que se les provee recursos, se establecen más como beneficiarios/favorecidos que como ciudadanos usuarios de derechos. ${ }^{17}$ La intervención, interpretada como donación, puede contribuir también a afirmar las condiciones de desigualdad en tanto otorga poder a los donantes y confirma su situación de no vulnerabilidad. Se establece entonces una conexión entre prodigalidad y servilismo; cuando se dona, se considera al otro como carente y desigual y, al mismo tiempo, se constituye un medio para la obtención de poder. La donación se convierte así en un peligroso vehículo que reproduce el servilismo y fortalece el poder de los donantes, independientemente de las razones que inspiren sus iniciativas.

\section{Discurso 2}

\section{El trabajo en el fortalecimiento de vínculos sociales}

Fisura 3: Desconocimiento de aspectos biográficos

Idealmente las intervenciones se ejecutan con el objeto de resolver situaciones de exclusión que, a su vez, se manifiestan en la insatisfacción de seguridades y necesidades sociales. Estas necesidades pueden clasificarse en dos tipos: en principio existirían unas necesidades básicas, universales u objetivas y, en segundo lugar, otras específicas, elaboradas o subjetivas. ${ }^{18}$ Esto sitúa a la intervención en una tensión respecto a los criterios que deben regular la selección de las necesidades a atender. Por lo general, las intervenciones optan por el trabajo sobre necesidades objetivas de gran escala ${ }^{19}$ lo que limita la consideración de situaciones individuales. Si bien este hecho ubica a la ISC en la ruta de la superación de grandes problemas sociales, se desconocen variables ligadas a comportamientos y actitudes que efectivamente contribuyen a explicar problemas sociales a partir de las trayectorias de vida.

\footnotetext{
${ }^{17}$ La intervención suele ratificar esta condición de donación haciendo énfasis en la ejecución pública de rituales de apertura y celebración de ciertos procesos que, como la inauguración del proyecto o actividades festivas para conmemorar la recepción de recursos, se inscriben como regalos en el imaginario social de la población receptora.

${ }^{18}$ Sánchez Vidal, Alipio, Ética de la intervención social, Paidós, Barcelona, 1999.

${ }^{19}$ Es importante pensar, por ejemplo, cómo las grandes estrategias del BID o del Banco Mundial se orientan en la erradicación de la pobreza en países subdesarrollados como necesidad básica que afecta a un alto porcentaje de la sociedad.
} 
Por otro lado, la intervención que se enfoca en la superación de necesidades subjetivas, identificadas como propias por el sujeto interrogado, no pueden comprenderse por fuera de él: son específicas y particulares a cada grupo humano. En este caso, la intervención busca ayudar al individuo a expresar y buscar satisfactores de necesidades que, eventualmente, pueden desencadenar procesos de orden colectivo. Esto indica que la relación entre necesidades colectivas y subjetivas no es excluyente. Las intervenciones sociales pueden generar procesos en los que los diferentes tipos de necesidades se superponen y complementan en el marco de procesos de concertación, explicitación e intencionalidad, comunes a todo proceso de intervención y que requieren de la negociación intersubjetiva entre actores sociales. En la acción sobre necesidades colectivas, la congruencia e intercambio de subjetividades es menor y las intervenciones giran alrededor de objetivos definidos previamente por el interventor. En los procesos de intervención individual, en cambio, se considera en la definición de objetivos las subjetividades de los sujetos y de su grupo íntimo identitario.

En la relación entre necesidades colectivas y subjetivas se evidencia una fisura clave para pensar los procesos de inserción a redes y el trabajo sobre fortalecimiento de vínculos sociales adelantado por la ISC. La dificultad en este caso radica en que no se encuentran puntos intermedios y complementarios en los que se atienda simultáneamente a los excluidos desde dimensiones macro y micro; éstos suelen ser intervenidos desde factores colectivos que, como la necesidad de integración al mundo del trabajo, no recogen sus necesidades, talentos ${ }^{20}$ y expectativas individuales. De esta manera las intervenciones consideran al sujeto excluido desde sus necesidades básicas y, siguiendo esta consideración, pretenden facilitar el acceso a derechos ciudadanos. Los procesos de formación para el empleo, por ejemplo, ilustran cómo, con el objeto de integrar a sujetos sociales a dinámicas económicas, se los capacita para el desarrollo de actividades con las que no necesariamente se identifican de manera subjetiva.

Fisura 4: Integración a redes de movilidad limitada

El fortalecimiento de vínculos y redes comunitarias permite reconocer y operar sobre problemas comunes en tanto facilita, entre otros, procesos como la identificación

\footnotetext{
${ }^{20}$ A este tema se refiere Richard Sennett en El respeto. Sobre la dignidad del hombre en un mundo de desiguldad, Anagrama, Barcelona, 2003.
} 
de líderes, la circulación de información importante para la movilización, la consolidación de niveles de organización necesarios para la resolución de problemas comunes y el robustecimiento de subjetividades e intersubjetividades en medio del reconocimiento de limitaciones y potencialidades colectivas e individuales. Sin embargo, a estos procesos de fortalecimiento de vínculos entre pares es posible cuestionarles su gestión al margen de la realidad económica y política. La exclusión, como ha sido mencionada previamente, es abordada sobre todo como un asunto individualista y comunitario, sin conexión con las realidades estructurales que la explican. Autores como Rosanvallón, ${ }^{21}$ por ejemplo, avalan esta tendencia cuando sostienen que la exclusión no es un nuevo problema social sino una "manera de describir las dificultades para establecer solidaridades sea de los individuos o sea de los grupos sociales”. En este sentido, serían “variables de comportamiento, y en particular de actitud con el trabajo, las que en fin explican mejor porqué las trayectorias de inserción son muy rápidas para algunos, muy entrecortadas para otros, muy inestables para muchos (...) Lo importante es, en primer lugar, analizar con claridad la naturaleza de las trayectorias que conducen a las situaciones de exclusión en tanto éstas son cada vez las resultantes de un proceso particular. De donde surge, por lo demás, la nueva importancia de las nociones de precariedad y vulnerabilidad”. ${ }^{22}$ Esto incide en la promoción de la solidaridad y participación sectorial que la ISC pretende gestionar con el objeto de integrar a sujetos en las dinámicas de ciudadanía plena, trabajo o intercambio económico, por medio de un ejercicio de formación en competencias comunitarias e individuales. Así, la ISC suele abordar los problemas de no ingreso al mercado y al mundo del trabajo desde un enfoque prioritariamente individual y comunitario, sin referencias de tipo causal. De acuerdo con esto, no sería la pertenencia a una categoría o sector social la que originaría la situación de desfavorecimiento, sino que habría un conjunto disperso y heterogéneo de individuos con el factor común de no poder insertarse en el mercado para resolver sus problemas de subsistencia y desarrollo personal. De forma consecuente con lo anterior, la ISC no reconoce en el sistema social las imperfecciones causantes de la pobreza y la marginación por lo que centra su labor al ámbito limitado de lo comunitario y lo comportamental.

Los logros apenas parciales de estas iniciativas, se explican en tanto su acción es también parcial y restringida: al ignorar demandas económicas y sociales, que superan

\footnotetext{
${ }^{21}$ Rosanvallón, Pierre, La nueva cuestión social, Manantial, Buenos Aires, 1995.

${ }^{22}$ Duschatzky, Silvia (compiladora), Tutelados y Asistidos. Paidós, Buenos Aires, 2000. (Prólogo, p. 23 ).
} 
el ámbito de lo comunitario, estas iniciativas consiguen mantener un equilibrio que contiene las contradicciones sociales sin hacerlas visibles y, por lo tanto, coarta las posibilidades de cambio. Si se reconoce que la integración plena pasa por la expansión de vínculos y posibilidades de movilidad social, es posible entender que el fortalecimiento de vínculos entre pares reduce el grado de movilidad. Las redes sociales establecidas entre pares marginados se caracterizan por su débil poder de influencia y participación ciudadana y económica. Así, no se fortalecen con los desafiliados

procesos de inserción reales: se les vincula a redes o a prácticas laborales que confirman su identidad deficitaria.

En conclusión, esta fisura hace evidente que si bien es incuestionable la importancia de producir una ciudadanía activa y recíproca, lo cierto es que la intervención hoy, divorciada del problema de distribución de la riqueza y de la responsabilidad estatal, estructura y ejecuta programas que apelan a la solidaridad de los grupos comunitarios que pueden terminar generando no sólo mayores dependencias sino procesos de naturalización de las desigualdades sociales y ciudadanas.

\section{Discurso 3}

\section{La focalización como estrategia de intervención}

Fisura 5: Ausencia de un proyecto integrador

Tal como se ha señalado, las intervenciones sociales son ejecutadas por organismos del Estado o por el tercer sector. Frente al desmantelamiento de las otrora seguridades sociales estatales, emergen con fuerza nuevas acciones desde instituciones que apuestan por la provisión de algunos servicios sociales predeterminados y ligados, en primera instancia a decisiones políticas e ideológicas. Desde el tercer sector y dado su marco de acción limitado, las intervenciones se realizan de manera segmentada, es decir, no se atiende a la población en general sino a aquellos que, dada una carencia específica, pueden ser incluidos como un grupo beneficiario o grupo de riesgo. Estas segmentaciones no necesariamente corresponden con la noción de clase social así que, además de la estratificación por ingreso socioeconómico, aparecen en el panorama de la intervención grupos poblacionales que comparten rasgos etarios, culturales, de salud, 
entre otros. Las intervenciones desde el tercer sector sobre estos grupos no tienen la pretensión de proveer seguridades sociales básicas.

Así, la intervención se presenta como una contracara del mercado: mientras éste propende cada vez más por mayor desterritorialización, es decir, por un intercambio y movilidad más allá del territorio físico y cultural, la intervención implica localización y sectorización; los integrados gozan de un intercambio cultural, económico y simbólico amplio, que supera los límites de lo local y nacional. A los intervenidos se los invita a trabajar, en cambio, en lo local y en el territorio próximo.

La focalización no se produce sin embargo sólo por un enfoque sectorial o territorial de la intervención, sino también por la atención fragmentada, y a través de proyectos de corto plazo, sobre necesidades de grupos objetivos. Al respecto, la focalización responde a las características de la atención social en el mundo contemporáneo que implican también un debilitamiento de las políticas universalizantes y de pérdida de sentido de ciertas utopías comunes. En este último punto, es posible determinar una diferencia entre antigua intervención ejercida desde el Estado y la intervención contemporánea. La intervención ejercida por el Estado, en forma de asistencia social, pretendía neutralizar las diferencias sociales con el objeto de integrar. La intervención contemporánea, en cambio, busca fragmentarse y focalizarse en las diferencias no siempre con pretensiones de integración pero sí de inclusión. La política de la focalización se hace visible, por un lado, como una idea de democratización, emprendida por la ISC, en tanto pareciera reconocer la heterogeneidad de proyectos e identidades particulares $\mathrm{y}$, por otro, como un ejercicio de resistencia a políticas universalizantes que aplastan las características y necesidades particulares de los grupos sociales. La fisura expuesta en este discurso se presenta en el hecho de que si bien la intervención expresa los errores de las políticas universalizantes, también se hace visible la ausencia de políticas integradoras. Se constituye en una intervención "sin horizonte normativo, sin fundamentos solidarios, invadida de ritualización, sin imaginación radical”. ${ }^{23}$ Si a esto se suma el hecho de que las intervenciones se efectúan bajo la lógica de proyectos de corto plazo, es posible comprender que una integración que supere los procesos sectoriales se diluye no sólo como política sino como posibilidad técnica. La eficacia de la gestión se comprueba a través del uso de procesos técnicos

\footnotetext{
${ }^{23}$ Boltanski, Luc, El Nuevo Espíritu del Capitalismo, Madrid, Ediciones Akal, 2000.
} 
evaluativos ligados a indicadores de resultados e impactos que, además de limitar la sostenibilidad, convierten los planes de intervención en respuestas coyunturales a urgencias sociales, determinadas por mediciones estadísticas.

Esta situación afecta los procesos de formación de ciudadanías plenas. Como se ha expuesto con anterioridad, la idea de ciudadanía plena en el mundo contemporáneo atañe a la condición de aquellos ciudadanos en capacidad de cumplir con deberes sociales de autosostenimiento y de acceder a derechos. Esta idea no estaría recogiendo a los excluidos como ciudadanos o, más bien, estaría configurando unas formas anómalas y débiles de ejercer ciudadanía. $^{24}$ Las políticas focalizantes, al afianzar la fragmentación social, fundan o refuerzan sectores de ciudadanos anómalos dada su dificultad para producir situaciones de autosostenimiento. Emerge entonces una paradoja: la condición de excluido indica el no acceso a una ciudadanía plena, en tanto los excluidos no cumplen totalmente con deberes sociales derivados de su capacidad de proveerse seguridad social. La ISC atiende a los sujetos intervenidos por su condición de exclusión. Esto implica que una de las condiciones no explícitas del sujeto intervenido es su no acceso a una ciudadanía plena. Así, aunque la ISC pretende generar procesos de inserción, en sus acciones puede convertirse en un poderoso dispositivo de afirmación de la situación de exclusión y de determinación de límites entre ciudadanos plenos y ciudadanos que no lo son.

\section{Conclusiones}

Han sido descritas hasta el momento cinco fisuras claves de los discursos que, desde la ISC, pretenden lograr en la población excluida la integración y autogestión de redes sociales. En resumen, se ha cuestionado la capacidad de la intervención para generar procesos de integración plena y se han descrito argumentos que incluso aseguran que las acciones de intervención podrían ratificar los procesos de exclusión. Si bien estos cuestionamientos no niegan los alcances de los procesos de intervención social, sí ofrecen otras perspectivas de sus efectos. Las conclusiones presentadas a continuación atraviesan los discursos sobre autonomía, fortalecimiento de vínculos sociales y prácticas de focalización y recogen los argumentos centrales expuestos en las fisuras

\footnotetext{
${ }^{24}$ Carballeda, Alfredo, La intervención en lo social. Exclusión e integración en los nuevos escenarios sociales, Paidós, Buenos Aires, 2002.
} 
descritas. Más que un conjunto de conclusiones definitivas, se presentarán algunos escenarios estratégicos para la discusión sobre la ISC y se ofrecen pistas que señalan caminos que permiten repensar la intervención a la luz de las necesidades de inclusión social.

1. Si se reconoce la relación, en apariencia inevitable, entre asistencia social y capitalismo, entre asistencia social y orden establecido, habría que moderar las expectativas sobre los procesos de intervención como motor de cambio y transformación en las estructuras sociales. Al respecto, es necesario partir de que si bien la intervención como ha sido concebida no va a erradicar la exclusión, podría evitar la legitimación discursos que simplifican la condición de los excluidos. Esto exige la producción de discursos más complejos sobre las relaciones entre exclusión y estructuras dominantes. En este sentido la demanda al mundo académico y a las ciencias sociales es evidente: se requiere producir nuevos discursos en los que la exclusión aparezca articulada a una dinámica social que la produce y se hagan visibles los mecanismos de esta dinámica social. En otras palabras, se requiere que se evidencie más la exclusión como un problema social que no compete sólo a los que la padecen y a sus trayectorias biográficas, sino que implica una cierta responsabilidad colectiva.

2. Por otro lado, si bien es inevitable reconocer que la autogestión y la promoción de la autonomía constituyen procesos claves en la superación de la exclusión, éstas no pueden fortalecerse sólo desde la generación de vínculos comunitarios, al margen de demandas sociales de mayor impacto. Si se define la autonomía como la posibilidad que tienen los actores de expandir su libertad de elección y acción, lo que implica incrementar la propia autoridad y control sobre los recursos y las decisiones que afectan su vida, se identifica que ésta no podría ejercerse sin una apuesta por la exigencia y demanda social al Estado. El trabajo que en este sentido debería emprender la ISC implica plantear una nueva relación entre individuo y sociedad. Hasta el momento la integración se ha pensado desde la perspectiva de la reciprocidad, esto es, desde la autogestión como modo de potenciar el cumplimiento de deberes por parte de los excluidos. Esta perspectiva es, sin embargo, insuficiente en tanto no demanda del Estado la garantía de medios que hagan plenamente posible este ejercicio de la 
autonomía. La intervención, al margen de sus relaciones con el Estado, debe considerar la movilización por la demanda social como una de las piezas claves que conforman el fortalecimiento de una autonomía plena. Sólo a través de un trabajo de este tipo es posible debilitar la idea de que los sujetos intervenidos son, más que actores responsables de su propio destino, beneficiarios y consumidores de programas sociales.

Frente a la intervención fragmentada y focalizada, carente de horizontes colectivos, se hace necesario movilizar la constitución de escenarios de trabajo, formas de solidaridad y desarrollo de ciudadanía que promuevan procesos de inclusión comunes a los ciudadanos en su diversidad biográfica y cultural. Esto exige, también, configurar un tipo de ciudadano intervenido no estigmatizado, a través de categorizaciones y acciones que no convaliden niveles ni escenarios desiguales de ciudadanía y que apunten a construir discursos inspirados en horizontes comunes. La apuesta en este sentido es por una intervención que considere las necesidades colectivas y subjetivas de los excluidos y que, desde este lugar, se piense la generación de planes y políticas para la inclusión y cohesión social plena.

\section{Bibliografía}

Aguilar, José María y Ezequiel Ander-Egg, Administración de Programas de Trabajo Social, Humanitas, Argentina, 1991.

Alvira, Francisco, Metodología de la Evaluación de Programas: Un Enfoque Práctico. Lumen, Buenos Aires, 1997.

Ander-Egg, Ezequiel, Evaluación de Programas de Trabajo Social, Humanitas, Argentina, 1984.

Ander-Egg, Ezequiel, Métodos y Prácticas. Desarrollo de la Comunidad, Humanitas, Argentina, 1982.

Ander-Egg, Ezequiel, Reconceptualización del Servicio Social, Humanitas, Argentina, 1976. 
Banco Mundial, Hacia una estrategia de desarrollo de ciudad en Cali, Banco Mundial, Washington D.C., 2002.

Bejarano, Enrique, et al., Intervención Psicosocial, Editorial Popular, Madrid, 1993.

Boltansky, Luc, El Nuevo Espíritu del Capitalismo, Madrid, Ediciones Akal S.A., 2000.

Briones, Guillermo, Evaluación de Programas Sociales, Trillas, México, 1999.

Carballeda, Alfredo, La intervención en lo social: exclusión en integración en los nuevos escenarios sociales, Paidós, México, 2001.

Carranza, Elías, "Políticas públicas en materia de seguridad de los habitantes de América Latina”, Nueva Sociedad, No.191, Caracas, 2000.

Castel, Robert, Las metamorfosis de la cuestión social: Una crónica del salariado, Paidós, Argentina, 1997.

Castro Carvajal, Beatriz, Caridad y Beneficencia en un Contexto de Pobreza: Colombia, 1870-1930, CIDSE, Cali, 2001.

Corporación Colombiana de Estudios Antropológicos para el Desarrollo (CEAD), Gobernabilidad, política pública y gestión pública, Bogotá, 2001.

Corvalán, Javier, "Cambios y permanencias en la intervención social en Chile. El discurso de las ONG y del Estado en las décadas del 80 y del 90", Tesis de Doctorado, Departamento de Ciencias Políticas y Sociales, Universidad Católica de Lovaina, Bélgica, 1996.

Cunill Grau, Nuria y Sonia Ospina Bozzi, "La Evaluación de Resultados para una Gestión Pública Moderna y Democrática. Experiencias Latinoamericanas”, en: Evaluación de Resultados para una Gestión Pública Moderna y Democrática. Experiencias Latinoamericanas, CLAD, Caracas, 2003.

De Robertis, Cristina, Metodología de la intervención en trabajo social, Editorial Ateneo. Buenos Aires, 1988.

Duschatzky, Silvia (compiladora), Tutelados y Asistidos, Paidós, Buenos Aires, 2000.

Garnier, Leonardo, "La Inversión Social: El Costo de las Oportunidades Perdidas", Centro de Desarrollo y asistencia Técnica en Tecnología para la Organización Pública (TOP), Buenos Aires, 1999, en: http://www.top.org.ar/publicac.htm.

Gough, Ian, Economía Política del Estado de Bienestar, H. Blum Ediciones, Madrid, 1982.

Gracia Fuster, Enrique, El Apoyo Social en la Intervención Comunitaria, Paidós, Barcelona. 1997. 
Hintze, Jorge, Administración de Estructuras Organizativas, Centro de Desarrollo y Asistencia Técnica en Tecnología para la Organización Pública (TOP), Buenos Aires, 1999.

Hintze, Susana, "Reflexiones sobre el Conflicto y la Participación en la Evaluación de Políticas Sociales”, Reforma y Democracia, No. 21 de CLAD, Caracas, 2001.

Kinersman, Natalio, et al., El Método: Intervención Transformadora, Humanitas, Buenos Aires, 1984.

Lazarsfeld, Paul, William Sewell y Harold Wilensky (compiladores), Planificación Sociológica de los Problemas Sociales, Centro Regional de Ayuda Técnica, México, 1971

Moe, Ferry, Sebastián Saiegh y Mariano Tommasi, "La Teoría Positiva de la Burocracia Pública”, en: La Nueva Economía Política: Racionalidad e Instituciones, Eudeba, Argentina, 1998.

Niremberg, O., I. Brawerman y V. Ruiz, Programación y Evaluación de Proyectos Sociales, Paidós, Buenos Aires, 2003.

Oszlak, Oscar, "Estado y Sociedad: Las Nuevas Fronteras”, en: El Rediseño del Perfil del Estado, Fondo de Cultura Económica, México, 1994.

Oszlak, Oscar, "Monitoring, Control and Evaluation in the Argentine Public Administration”, Informe de Investigación, Banco Interamericano para el Desarrollo, Washington D.C., 1987.

Ponticelli, María Del Pilar, Modelos teóricos del trabajo social, Editorial Lumen/ Humanitas, Buenos Aires, 1998.

Quintero Uribe, Víctor Manuel, Evaluación de Proyectos Sociales: Construcción de Indicadores Fundación para la Educación Superior, FES, Cali, 1995

Quintero Uribe, Víctor Manuel, y Harold Enrique Banguero, Los Proyectos Sociales: Guía para su Evaluación y Análisis de Factibilidad Colombia, Instituto FES de Liderazgo, Cali. 1991.

Rosanvallón, Pierre, La Nueva Cuestión Social, Manantial, Buenos Aires, 1995.

Sallenave, Jean Paul, Gerencia y Planeación Estratégica, Norma, Bogotá, 2002.

Sánchez, Alipio, Ética de la intervención social, Paidós, Barcelona, 1999.

Sánchez, Alipio y G. Musitu (compiladores), Intervención Comunitaria: Aspectos Científicos, Técnicos y Valorativos, EUB. Barcelona, 1996.

San Juan, César, Intervención Psicosocial, Anthropos, Bogotá, 1996.

Sen, Amartya, Desarrollo y Libertad, Planeta, Bogotá, 2000. 
Sennett, Richard, El respeto. Sobre la dignidad del hombre en un mundo de desigualdad, Anagrama, Barcelona, 2003.

Sennett, Richard, Nuevo Examen de la Desigualdad, Alianza, Madrid, 1995.

Touraine, Alain, Sociología de la Acción, Ariel, Barcelona, 1969.

Touraine, Alain, “Introducción al Método de la Intervención Sociológica”, Estudios Sociológicos, Vol. 4, No. 11, mayo-agosto, 1986, Colmes, México.

Tulchin, Joseph y Fagan Graig, "Perfil actual de la seguridad ciudadana e impacto en la gobernabilidad democrática. Aportes desde Latinoamérica”, en: Bobea, Lilian (ed), Entre el crimen y el castigo. Seguridad ciudadana y control democrático en América Latina y el Caribe, Nueva Sociedad, 2003.

Zamosc, León, Campesinos y Sociólogos: Reflexiones sobre Dos Experiencias de Investigación Activa en Colombia, VV.AA, Investigación, Acción Participativa en Colombia. Bogotá. Editorial Punta de Lanza y Fundación Foro, 1987. 